\title{
PROFILAXIA INTERMITENTE COM DIAZEPAM VIA ORAL NA CONVULSÃO FEBRIL
}

\author{
ESTUDO DE 82 CASOS \\ MARISTELA COSTA * EDNEA A. SILVA**, \\ ANNA E. S. MENDES DA SILVA**, MARILISA M. GUERREIRO** *
}

\begin{abstract}
RESUMO - Apresentamos os resultados do uso de diazepam intermitente na profilaxia da convulsão febril (CF). Acompanhamos 82 pacientes com idade entre 3 meses e 5 anos por período médio de 15,8 meses. Dezoito crianças ( $21,95 \%$ da casuística) apresentaram efeitos colaterais transitórios que não limitaram o uso da medicação. Houve recorrência em $26 \%$ dos casos. Concluimos que o diazepam, via oral, é eficaz para prevenir recorrência de CF. Ele apresenta fácil manuseio e boa tolerabilidade. Sugerimos o seu uso em crianças que apresentem algum fator de risco preditivo para recorrência.
\end{abstract}

PALAVRAS-CHAVE: convulsão febril, diazepam.

\section{Intermittent prophylaxis in febrile seizures with oral diazepam: study of 82 cases}

ABSTRACT - We present our results of intermittent prophylaxis with oral diazepam in febrile seizures. We treated 82 patients aged between 3 months and 5 years. They have had simple or complex febrile seizures. Recurrence occurred in 22 patients $(26 \%)$, none had a long-lasting febrile convulsion. Transient side effects occurred in $21.95 \%$ of the cases. We conclude that diazepam is a safe and effective drug for prophylaxis of febrile seizures when used as soon as any sign of illness appears. We suggest, however, that the administration of the drug should be indicated if the child presents at least one consistent predictor of risk of recurrent febrile seizures.

KEY WORDS: febrile seizures, diazepam.

Convulsão febril (CF) ocorre na infância, geralmente entre os 3 meses e 5 anos de idade, associada a febre, na ausência de infecção intracraniana ou de outra causa neurológica definida, excluindo-se as crianças que tenham tido previamente convulsões afebris ${ }^{8,20,23}$. A primeira CF ocorre em média aos 23 meses podendo ser de 2 tipos: simples (uma única crisc tônico-clônica generalizada com duração geralmente ao redor de 5 minutos) e complexa ou complicada (crises focais e/ou com duração maior que 15 minutos e/ou se recorrer em menos de 24 horas) $)^{23}$. A CF é entidade com caráter extremamente benigno e, por isso, 3 questões têm sido enfocadas na literatura: qual é o risco de recorrência, qual é a melhor opção para profilaxia e quando esta estaria indicada. Discutiremos cada tópico mais adiante. Quando se opta pela profilaxia, esta pode ser feita de 2 maneiras: profilaxia contínua com fenobarbital ${ }^{1,7,12,16,30}$ ou com valproato ${ }^{35}$ e profilaxia intermitente com diazepam ${ }^{6,13-}$ 16,32,33, via oral ou retal. As drogas utilizadas para a profilaxia contínua apresentam importantes efeitos colaterais ${ }^{1,7,8,26}$, enquanto que o diazepam apresenta eficácia semelhante, sem efeitos colaterais que limitem a sua utilização ${ }^{32}$.

Estudo realizado na Disciplina de Neurologia Infantil do Departamento de Neurologia da Faculdade de Ciências Médicas (FCM) da Universidade Estadual de Campinas (UNICAMP): *Residente de Neurologia Infantil; ** Pós-Graduanda em Neurociências; *** Professor Assistente Doutor. Aceite: 16-janeiro-1996. 
Este estudo tem a finalidade de mostrar a nossa experiência com o uso do diazepam via oral na profilaxia da CF e divulgar os resultados de nossa avaliação, em continuidade ao registro anteriormente feito na fase preliminar ${ }^{10}$.

\section{MATERIAL E MÉTODOS}

Participaram deste estudo todas as crianças entre 3 meses e 5 anos que apresentaram CF e que procuraram atendimento médico no Ambulatório de Epilepsia Infantil do Hospital das Clínicas da UNICAMP, no periodo de janeiro de 1990 a abril de 1994 . Todas as crianças foram seguidas prospectivamente. Foram incluidas $\mathrm{CF}$ simples ou complicadas, independentemente dos antecedentes pessoais ou neurológicos, e excluidas as crianças com crises afebris prévias.

Nos casos em que os pacientes já utilizavam profilaxia contínua, a familia era esclarecida sobre as vantagens da profilaxia intermitente e se concordassem, a troca das medicações era feita. A profilaxia intermitente com diazepam via oral foi indicada na dose de $0,3-0,5 \mathrm{mg} / \mathrm{kg} /$ dose em 2 tomadas ao dia, sempre que a criança apresentasse febre, mantendo-a por 24 horas após o último pico febril, a fim de se certificar de que o processo febril estava controlado. As familias foram orientadas para usar medicação antitérmica e a procurar o pediatra para esclarecer a etiologia da febre, bem como para utilizar medicação específica quando fosse necessário (como antibióticos e antiinflamatórios).

Nenhum exame complementar fez parte do protocolo. Os retornos foram agendados trimestralmente ou antes se houvesse necessidade.

\section{RESULTADOS}

Participaram do protocolo 110 crianças com idades variando de 4 meses a 5 anos e 2 meses; entretanto, 28 não aderiram ao tratamento. Foram acompanhadas, assim, 82 crianças por tempo médio de 15,8 meses (mínimo de 6 meses e máximo de 48 meses). Cinquenta e duas (63,4\%) eram do sexo masculino e $30(36,6 \%)$, do feminino.

A faixa etária por ocasião da primeira CF encontra-se exposta na Tabela 1; em 50 pacientes (62\%), a primeira CF ocorreu antes dos 18 meses.

Das 82 crianças, $56(68,3 \%)$ apresentaram CF simples e $26(31,7 \%)$, CF complicada.

A recorrência se deu em 22 pacientes (26\%), dos quais 12 apresentaram rápida ascenção da febre sem tempo hábil para o uso adequado do diazepam, 12 haviam manifestado a primeira CF antes de 18 meses de idade e 6 tinham antecedente familiar positivo para CF (Tabela 2). Finalmente, 6 crianças não tinham fator de risco preditivo para recorrência.

Levando-se em conta apenas esses 3 fatores de risco, ou seja, ascenção rápida da febre, idade inferior a 18 meses na primeira $\mathrm{CF}$ e antecedente familiar positivo para $\mathrm{CF}$, encontramos no grupo que apresentou recorrência: 9 crianças com 1 fator, $5 \operatorname{com} 2$ fatores e $2 \operatorname{com} 3$ fatores de risco para recorrência (Tabela 3).

Dezoito pacientes $(21,95 \%)$ apresentaram efeitos colaterais (Tabela 4$)$, todos de caráter transitório, não limitando o uso do diazepam via oral.

Tabela 1. Idade na primeira convulsão febril.

\begin{tabular}{ccc}
\hline $\begin{array}{c}\text { Idade em } \\
\text { meses }\end{array}$ & $\begin{array}{c}\text { Número de } \\
\text { crianças }\end{array}$ & $\begin{array}{c}\text { Porcentagem de } \\
\text { pacientes }\end{array}$ \\
$3-6$ & 23 & $28 \%$ \\
$6-12$ & 8 & $10 \%$ \\
$12-18$ & 19 & $23 \%$ \\
$18-24$ & 12 & $14,5 \%$ \\
$>24$ & 20 & $24,5 \%$ \\
Total & 82 & $100 \%$ \\
\hline
\end{tabular}

Tabela 2. Perfil de 22 crianças que apresentaram recorrência.

Fator de risco

Número de casos

Tempo de ascensão da febre $<1$ hora

Idade na primeira $\mathrm{CF}<18$ meses

História familiar positiva para $\mathrm{CF}$

Sem fator de risco 
Tabela 3. Fatores de risco acumulados.

\begin{tabular}{cc}
\hline $\begin{array}{c}\text { Número de } \\
\text { fatores de risco }\end{array}$ & $\begin{array}{c}\text { Número de } \\
\text { pacientes }\end{array}$ \\
\hline 1 & 9 \\
2 & 5 \\
3 & 2 \\
\hline
\end{tabular}

Tabela 4. Efeitos colaterais.

\begin{tabular}{lcc}
\hline Efeito colateral & $\begin{array}{c}\text { Número de } \\
\text { pacientes } \\
\text { (porcentagem) }\end{array}$ & $\begin{array}{c}\text { Porcentagem em } \\
\text { relaçäo ao número } \\
\text { total de 82 pacientes }\end{array}$ \\
\hline Agitação & $5(27,8 \%)$ & $6,09 \%$ \\
Sonolência & $11(61,1 \%)$ & $13,41 \%$ \\
Ataxia & $2(11,1 \%)$ & $2,43 \%$ \\
Total & $18(100 \%)$ & $21,95 \%$ \\
\hline
\end{tabular}

Entre os pacientes que apresentaram recorrência, não observamos qualquer convulsão de longa duração, status epilepticus, morte ou sequela neurológica.

$\mathrm{Na}$ evolução, uma criança apresentou uma crise afebril e outra, epilepsia, perfazendo $2,4 \%$ da casuística.

\section{DISCUSSÃO}

A CF apresenta alta incidência na população infantil, ocorrendo em 2 a $5 \%$ das crianças nos primeiros 5 anos de vida ${ }^{11,21}$, o que justifica a importância desse tema. No presente estudo encontramos prevalência de meninos $(52: 30)$, o que é concordante com a literatura ${ }^{20}$. A primeira CF ocorreu principalmente entre 12 e 18 meses, diferentemente dos achados de Millichap ${ }^{20}$ e Nelson e Ellenberg ${ }^{23}$ que observaram picos de ocorrência entre 18-22 meses e aos 23 meses, respectivamente. Dentre as 82 crianças, $31,7 \%$ apresentaram CF complicada, sendo que alguns estudos epidemiológicos apontam taxas próximas a $25 \%{ }^{27}$. A precocidade do pico de ocorrência da primeira CF e a maior frequência de CF complicada na presente casuística evidenciam que a nossa população não é representativa da população geral infantil, talvez pelo fato de este estudo ter sido desenvolvido em hospital universitário de referência.

Atualmente, a CF é entidade muito bem estudada e sempre é realçado seu caráter extremamente benigno, o que faz com que algumas questðes devam ser enfocadas ${ }^{27}$. Assim, o principal tópico a ser abordado é porque tratá-la, havendo aspectos a favor e contra a serem considerados:

- a ansiedade da familia perante a crise e o risco de a criança apresentar um trauma por ocasião da recorrência ${ }^{15,24}$;

- a possibilidade de recorrência - este sempre foi um dos aspectos mais estudados, estando hoje bem definidos os fatores de risco, a saber: parentes de primeiro grau com história de CF, primeira crise antes de 18 meses (a maior chance de recorrência ocorre entre 12 e 14 meses de sua vida, geralmente nos primeiros 6 meses após a CF inaugural), o grau da febre e o tempo de ascenção antes da primeira $\mathrm{CF}^{2,3,15,21,29}$ (Tabela 5). Knudsen ${ }^{14}$ levantou, ainda, 3 outros fatores: CF complicada, antecedente familiar para epilepsia e a presença da criança em creche. Destes 3 fatores, apenas antecedente familiar para epilepsia parece realmente ser fator preditivo importante para recorrência, conforme demonstrado por Offringa ${ }^{27,28}$ e Rantala ${ }^{31}$. Apesar de alguns dos fatores apontados por Knudsen'4 não serem aparentemente relevantes, este autor expôs a idéia de que a somação dos fatores preditivos implica numa maior chance de recorrência, a qual aumenta proporcionalmente ao número de fatores preditivos presentes. No presente estudo houve alta taxa de recorrência, $26 \%$, possivelmente justificada pelo fato da maioria destas 22 crianças apresentar pelo menos um fator de risco (Tabela 3). Para o levantamento dos fatores de risco presentes na populaçăo deste estudo, levamos em consideração aqueles mais comumente referidos ${ }^{23.315 .21 .25 .29}$ expostos na Tabela 2. Entretanto, novos trabalhos ${ }^{27,28,31}$, acrescentaram alguns dados àqueles já relevantes e a conclusăo é que, no momento, são enfatizados os fatores apresentados na Tabela 5. Os nossos casos evidenciaram 
Tabela 5. Fatores de risco preditivos para recorrência de CF*.

Parentes de primeiro grau com história familiar positiva para convulsão febril ou epilepsia

Idade na primeira convulsão febril (< 18 meses)

Temperatura no momento da CF e duração da febre antes da CF

*Conforme referências 27,28 e 31

que pode ocorrer falha do tratamento profilático com diazepam, quando não há tempo para medicar a criança por causa da subida rápida da temperatura;

- a possibilidade de ocorrer uma convulsão de longa duração na recorrência - embora pequena $^{15,28}$, esta possibilidade nos leva a mencionar a provável associação existente entre convulsão febril e esclerose mesial hipocampal. Discute-se o que seria causa ou consequência e se realmente existe relação entre elas ${ }^{5,17-19,27}$. Entre os pacientes que tiveram recorrência na vigência de profilaxia com diazepam, não observamos qualquer convulsão de longa duração, o que já havia sido constatado por Knudsen ${ }^{15}$. Teremos, entretanto, que aumentar nossa casuística antes de tirarmos qualquer conclusão, principalmente porque o papel da convulsão prolongada ainda não está claro;

- o aparecimento de epilepsia posterior - é uma possibilidade associada a CF complicada, alteração no exame neurológico e história familiar de epilepsia. Não há evidências de que a profilaxia medicamentosa mude esse perfil. O número de recorrências, talvez, também esteja relacionado com posterior evolução para epilepsia ${ }^{9,15,21,23,34}$. Houve pequena incidência de epilepsia posterior entre nossos casos, o que provavelmente se deve ao curto periodo de acompanhamento;

- morte ou sequela neurológica permanente - são eventos relacionados com status epilepticus, o qual vem diminuindo muito com a melhora do atendimento de emergência. Assim como foi referido no estudo populacional de Nelson e Ellenberg ${ }^{15,18}$, não houve qualquer morte ou déficit neurológico definitivo em nosso estudo;

- alteração das funções corticais superiores - estas funçôes foram estudadas em crianças na faixa escolar entre 7 e 11 anos, não se detectando diferenças entre as que apresentaram CF e as do grupo controle ${ }^{23}$. Não há estudos, contudo, visando à detecção de alterações sutis ou que avaliem toda a vida escolar ${ }^{15}$.

Todos estes aspectos realçam o caráter benigno da CF e diante de tantos argumentos muitos autores acreditam que não se precisa considerar a necessidade de tratamento ${ }^{3,4,8}$. Entretanto, entre os que consideram que devem ser tratadas apenas as crianças com fator preditivo para recorrência, aparece a dúvida de qual seria a melhor escolha terapêutica: a profilaxia contínua com fenobarbital ${ }^{12,30,35}$, mais raramente com valproato ${ }^{35}$, ou a intermitente com diazepam ${ }^{6,13-16,32,33}$. A profilaxia com fenobarbital apresenta efeitos colaterais inconvenientes que ocorrem em até $40 \%$ dos usuários: hiperatividade, irritabilidade, distúrbio do sono ${ }^{15,16,17,30}$. Além disso, embora geralmente seja considerada eficaz, há estudos que sugerem que o fenobarbital não é um bom agente para a profilaxia da $\mathrm{CF}^{7,26}$. Ainda, deve ser analisado o aparente risco de decréscimo do quociente da inteligência (QI), conforme demonstrado em estudo randomizado ${ }^{8}$. Com o valproato, é referido o risco de hepatite fulminante, efeito colateral raro, mas que limita seu uso em crianças pequenas, principalmente numa entidade tão benigna como a $\mathrm{CF}^{3}$. Assim, o uso do diazepam pareceu-nos a opção mais prudente para a profilaxia da $C F$.

A tolerabilidade à droga foi boa, sendo que $21,95 \%$ dos pacientes apresentaram efeitos colaterais de leve a moderada intensidade, porém transitórios, que não impossibilitaram o uso da medicação. Esses dados vão de encontro aos da literatura ${ }^{10,15,32}$.

Concluímos que o diazepam oral é eficaz para prevenir recorrência de CF e é de boa tolerabilidade e de fácil manuseio, o que é discutível no caso da via retal. Sugerimos, entretanto, que 
seu uso seja restrito aos casos com algum fator de risco para recorrência e que a família seja orientada para que a profilaxia seja iniciada a qualquer sinal de adoecimento da criança.

\section{REFERÊNCIAS}

1. Addy DP. Prophylaxis and febrile convulsions. Arch Dis Child 1981;56:81-83.

2. Berg AT, Shinnar S, Hauser WA, Leventhal JM. Predictors of recurrent febrile seizures: a metaanalytic review. J Pediatrics 1990;116:329-337.

3. Berg AT, Shinnar S, Hauser WA, Alemany M, Shapiro ED, Salomon ME, Crain EF. A prospective study of recurrent febrile seizures. N Engl J Med 1992;327:1122-1127.

4. Camfield P, Camfield C. Diazepam to prevent febrile seizures. N Engl J Med 1993;329:2034.

5. Cendes F, Andermann F, Dubeau F, Gloor P, Evans A, Jones-Gotman M, Olivier A, Andermann E, Robitaille Y, LopesCendes I, Peters T, Melanson D. Early childhood prolonged febrile convulsions, atrophy and sclerosis of mesial structures, and temporal lobe epilepsy: an MRI volumetric study. Neurology 1993;43:1083-1087.

6. Dianese G. Prophylaxis of febrile convulsions: searching for the best. Arch Dis Child 1986;61:621-622.

7. Farwell JR, Lee YJ, Hirtz DG, Sulzbacher SI, Ellenbery JH, Nelson KB. Phenobarbital for febrile seizures: effect on intelligence and on seizure recurrence. N Engl J Med 1990;322:364-369.

8. Freeman JM. Febrile seizures: a consensus of their significance, evolution, and treatment. Pediatrics 1980;66:1009-1012.

9. Freeman JM. The best medicine for febrile seizures. N Engl J Med 1992;327:1161-1162.

10. Guerreiro MM, Costa M, Bellomo MA, Sabino SH, Scotoni AE. Profilaxia intermitente na convulsão febril com diazepam via oral. Arq Neuropsiquiatr 1992;50:163-167.

11. Hauser WA. The natural history of febrile seizures. In Nelson KB, Ellenberg JH (eds). Febrile seizures. New York: Raven Press, 1981:5-17.

12. Herranz JL, Armijo JA, Arteago K. Effectiveness and toxicity of phenobarbital, primidone and sodium valproate in prevention of febrile convulsions controlled of plasma levels. Epilepsia 1984;25:89-95.

13. Knudsen FU. Effective short-term diazepam prophylaxis in febrile convulsions. J Pediatr 1985;106:487-490.

14. Knudsen FU. Recurrent risk after first febrile seizure and effect of short-term diazepam prophylaxis. Arch Dis Child 1985;60:1045-1049.

15. Knudsen FU. Intermittent diazepam prophylaxis in frebile convulsions. Acta Neurol Scand 1991;83 (Suppl 135):1-24.

16. Knudsen FU, Vestermark S. Prophylatic diazepam or phenobarbital in febrile convulsions: a prospective controlled study. Arch Dis Child 1978;53:660-663.

17. Kuks JBM, Fish DR, Stevens JM, Shorvon SD. Hippocampal sclerosis in epilepsy and childhood febrile seizures. Lancet 1993;342:1391-1394.

18. Maytal J, Shinnar S. Febrile status epilepticus. Pediatrics 1990;86:611-616.

19. Moshé SL. Seizures in developing brain. Neurology 1993;43(Suppl 5):S3-S7.

20. Millichap JG. The definition of febrile seizures. In: Nelson KL, Ellenberg JH (eds). Febrile seizures. New York: Raven Press, $1981 ; 1-3$.

21. Nelson KB. Can treatment of febrile seizures prevent subsequent epilepsy? In Nelson KB, Ellenberg JH (eds). Febrile seizures. New York: Raven Press, 1981:143-145.

22. Nelson KB, Ellenberg JH. Predictors of epilepsy in children who have experienced febrile seizures. N Engl J Med 1976;295:1029-1033.

23. Nelson KB, Ellenberg JH. Prognosis in children with febrile seizures. Pediatrics 1978;61:720-727.

24. Nelson $K B$, Ellenberg $J$. The role of recurrences in determining outcome in children with febrile seizures. In: Nelson $K B$, Ellenberg JH (eds). Febrile seizures: New York: Raven Press, 1981;19-25.

25. Nelson KB, Ellenberg JH. Prenatal and perinatal antecedent of febrile seizures. Ann Neurol 1990;27:127-131.

26. Newton RW. Randomized controlled trials of phenobarbitone and valproate in febrile convulsions. Arch Dis Child 1988, 63:1189-1191.

27. Offringa M. Seizures associated with fever: current management controversies. Sem Ped Neurol 1994;11:90-101.

28. Offringa M, Bossuyt P, Lubsen J, Ellenberg J, Nelson K, Knudsen F, Annegers J, El-Radhi A, Habbema J, Derksen-Lubsen G, Hauser W, Kurland L, Banajeh S, Larsen S. Risk factors for seizure recurrence in children with febrile seizures: a pooled analysis of individual patient data from five studies. J Pediatr 1994;124:574-584.

29. Offringa $M$ Derksen-Lubsen $G$, Bossuyt $P$, Lubsen J. Seizure recurrence after a firts febrile seizure: a multivariate approach. Dev Med Child Neurol 1992;34:15-24.

30 . Pilgaard S, Hansen J, Perregaard P. Prophylaxis against febrile convulsions with phenobarbital. Acta Pediatr Scand 1986;70:67-71.

31. Rantala H, Uhari M. Risk factors for recurrences of febrile convulsions. Acta Neurol Scand 1994;90:207-210.

32. Rosman NP, Colton T, Labazzo J, Gilbert PL, Gardella NB, Kaye EM, van Bennekom C, Winter MR. A controlled trial of diazepam administered during febrile illnesses to prevent recurrence of febrile seizures. N Engl J Med 1993;329:79-84.

33. Thorn 1. Prevention of recurrent febrile seizures: intermittent prophylaxis with diazepam compared with continuous treatment with phenobarbital. In Nelson KB, Ellenberg JH (eds). Febrile seizures. New York: Raven Press, 1981:119-126.

34. Verity CM, Golding G. Risk of epilepsy after febrile convulsions: a national cohort study. Br Med J 1991;303:373-376.

35. Wallace SJ. Prevention of recurrent febrile seizures using continuous prophylaxis: sodium valproate compared with phenobarbital. In Nelson KB, Ellenberg JH (eds). Febrile seizures. New York: Raven Press, 1981:135-142. 